\title{
Sensitive Detection and Early Prognostic Significance of p24 Antigen in Heat- Denatured Plasma of Human Immunodeficiency Virus Type 1-Infected Infants
}

\author{
J. Schüpbach, J. Böni, Z. Tomasik, J. Jendis, R. Seger, \\ C. Kind, and the Swiss Neonatal HIV Study Group*
}

\author{
Swiss National Center for Retroviruses, Institute of Medical Virology. \\ University of Zurich, and Division of Immunology and Hematology. \\ Children's University Hospital, Zurich; Division of Neonatology.
}

Kantonsspital, St. Gallen, Switzerland

\begin{abstract}
Immune complex formation causes underdetection of p24 antigen in human immunodeficiency virus (HIV) infection. Briefly boiling diluted plasma releases all complexed antigen, which can then be measured by some commercial assays. In a retrospective pediatric cohort study, the specificity of this procedure in 390 uninfected samples was $96.9 \%$ after initial testing and $100 \%$ after neutralization. Sensitivity among 125 postnatal infected samples was, at a detection of 2 $\mathrm{pg} / \mathrm{mL}, \mathbf{9 6 . 0} \%$ (97\% neutralizable) compared with $47.7 \%$ for regular antigen (76\% neutralizable), $96 \%$ for polymerase chain reaction, and $77 \%$ for viral culture. The high sensitivity and specificity of heat-denatured antigen was confirmed by prospectively testing 113 additional samples. Quantitative analysis of samples from infected infants showed low levels of p24 antigen in $29 \%$ of cord blood sera, a postnatal increase to levels that were during the first 6 months of life inversely associated with survival, and persistence of antigenemia thereafter independent of clinical status. Prevalence and antigen levels were significantly lower in mothers. The persistent antigenemia in children indicates that their immune systems cannot restrict HIV expression as efficiently as those of adults.
\end{abstract}

Detection of human immunodeficiency virus type 1 (HIV1) p24 antigen in early pediatric HIV infection has been of low sensitivity, due to the presence of usually high-titered maternal anti-p24 IgG antibodies, which lead to immune complex formation of the antigen [1-4]. Acid dissociation of immune complexes has resulted in improved antigen detection in plasma or serum of infected adults and children [511]. However, we recently showed that the acid-mediated release of small quantities of antigen from immune complexes is inefficient if antibodies to $\mathrm{p} 24$ antigen are present at very high titers [12]. In contrast, we have found that boiling diluted serum leads to the quantitative and irreversible release of immune-complexed HIV-1 p24 that can be readily measured with commercial assays that recognize heat-denatured antigen (HD-Ag). In a study of 50 sera from HIV-positive adults, this procedure was significantly superior to acid dissociation.

We used this simple system to analyze serum or plasma

Received 23 August 1993; revised 10 January 1994.

Presented in part: IXth International Conference on AIDS, Berlin, 6-11 June 1993 (abstract WS-B05-5).

Written informed consent was obtained from parents or legal guardians in accordance with the guidelines of the Swiss National Academy of Medical Sciences.

Financial support: Swiss Federal Office of Public Health.

Reprints or correspondence: Dr. J. Schüpbach, Swiss National Center for Retroviruses, Institute of Medical Virology, University of Zurich, Gloriastrasse 30, CH-8028 Zurich. Switzerland.

* Members are listed after the text.

The Journal of Infectious Diseases 1994;170:318-24 (C) 1994 by The University of Chicago. All rights reserved. $0022-1899 / 94 / 7002-0011 \$ 01.00$ samples of children born to HIV-1-infected mothers. We assessed the diagnostic sensitivity and specificity of the procedure in a cohort of well-characterized pediatric samples and virus expression in children with HIV-1 infection.

\section{Materials and Methods}

Patients and samples. Blood samples were from children born to HIV-1-seropositive mothers. All children were participants in the prospective Swiss Neonatal HIV Study, which has been maintaining a national data base since October 1986 [13]. None of the children had received specific antiretroviral treatment. EDTA-anticoagulated blood samples were collected from infants at birth (cord blood) and at 3- to 6-month intervals thereafter. A maternal serum sample was collected at delivery. An infant was considered infected if at least one of the following criteria was satisfied: positive IgG Western blot (WB) (defined below) after age 15 months, positive polymerase chain reaction (PCR) or viral culture or neutralizable antigen in $\geqslant 2$ different blood samples, or death from AIDS. The age at which symptoms were first recognized (CDC P-2) was defined as the age at onset of symptomatic HIV infection [14]. Children ruled uninfected included those with complete seroreversion and negativity by all tests for viral components (see below) and those with $\geqslant 2$ consecutive postnatal samples negative by PCR, provided that all other tests for viral components were also negative.

The retrospective blinded study for HD-Ag included all frozen serum or plasma samples still available from cohort children: 690 samples representing 305 (72.3\%) of the 422 infants. Samples were from 362 boys and 328 girls (ratio: 1.10). The ratio for the cohort was 193 to $229(0.84)$. The median age at which samples were obtained was 11 months (range, 0-95). Due to the study design, only few postnatal samples of very 
young infants were available, including 5 from the first and 7 from the second month. Samples from children with verified presence or absence of HIV infection were of particular interest: There were 142 (20.6\%) samples from 53 infected children. The mean age represented by these samples was 18 months (range, 0-92 months). A total of 390 samples (56.5\%) was from 134 uninfected children (mean age, 12 months; range, 0-95). For the remaining 157 samples (22.9\%), the status of infection was not resolved and the results are not described.

In addition to the above samples, 113 samples from cohort children were analyzed by HD-Ag and PCR in a prospective study conducted since August 1992. The age range represented by these samples was 0 to 111 months (median 11).

Antibody tests. All samples were prospectively tested for HIV-1 IgG by WB using strips from Biotech Research Laboratories (Rockville, MD), Bio-Rad Laboratories (Glattbrugg, Switzerland), or Diagnostic Biotechnology (Geneva). Results were interpreted on the basis of recommendations of the following organizations: the Centers for Disease Control and Prevention and the Association of State and Public Health Laboratory Directors, the Consortium for Retrovirus Serology Standardization, the American Red Cross, and the US Food and Drug Administration [15]. A sample was ruled WB-positive if it met criteria for positivity of at least three of the organizations.

Tests for viral components. Assays done prospectively included antigen tests with undenatured samples (UD-Ag), viral culture, and PCR. HD-Ag was tested retrospectively or prospectively. All testing was done blinded.

The Abbott HIV AG EIA (Abbott Diagnostics, WiesbadenDelkenheim, Germany) and later the Du Pont (Bad Nauheim, Germany) HIV-1 p24 core profile ELISA were used for UD-Ag testing of undiluted serum or plasma samples. Since 1990 (i.e.. in $55 \%$ of all samples evaluated for this study), the tests were done in duplicate. Positive samples were subjected to confirmatory neutralization, using the kits and procedures of the respective manufacturers.

Viral culture was done prospectively on ficoll-purified peripheral blood mononuclear cells (PBMC) cocultured with phytohemagglutinin-stimulated PBMC from HIV-negative blood donors [16]. The sole modification in the technique was that cultures were kept in 15-mL polyallomer tubes (Falcon; Becton Dickinson, Basel, Switzerland). Duplicate samples of supernatants were tested for UD-Ag twice a week. A positive result required the presence of antigen above the manufacturer's cutoff level in at least 2 consecutive samples.

For PCR, duplicates of $1-\mu \mathrm{g}$ samples of DNA extracted from ficoll-separated PBMC were amplified with primers from the HIV-1 gag and long terminal repeat regions. Amplified material was analyzed by a liquid hybridization procedure followed by resolution of the products on a denaturing gel as described [17]. The procedure can detect a single copy of HIV-1 DNA. A positive result required reaction with both primer regions and at least one in duplicate. DNA extraction and testing of all samples was done blindly. The specificity record of PCR in routine diagnostic applications in our laboratory was $>99.6 \%$ in 1990 (no false-positives among 287 negative control reactions) and $99.2 \%$ in both 1991 and 1992 (4/531 and 6/708 false-positive reac- tions, respectively). Accordingly, the probability of a false-positive result ( $\geqslant 3$ positive reactions/sample) was negligible $(.008)^{3}$. In fact, none of 172 negative control samples obtained and tested in 1992 had a false-positive result.

For HD-Ag testing, patient or control samples were diluted with 2 vol of a $0.5 \%$ solution of Triton X-100. The diluted samples were boiled for $5 \mathrm{~min}$ in 1.5-mL Eppendorf tubes on a dry heat block (DRI-BLOCK DB-2A; Techne, Cambridge, UK) and assayed in duplicate by the Du Pont HIV-I p24 core profile ELISA according to the manufacturer's instructions, except that incubation of serum or plasma samples was for $2 \mathrm{~h}$ at room temperature on a microplate shaker (Vary Shaker; Dynatech, Glattbrugg, Switzerland). Antigen standards of 3, 6, 12.5, 25, 50 , and $100 \mathrm{pg} / \mathrm{mL}$ in duplicate and an antigen-negative control in triplicate were included in all experiments as described [12]. Samples positive in screening, using a previously established cutoff of 0.020 absorbance units, were subjected to a confirmatory neutralization assay (Du Pont). The manufacturer's instructions were followed with the same exceptions as above.

Statistical analysis. Proportions were compared by $\chi^{2}$ test when the total number of samples was $\geqslant 40$. Fisher's exact test was used for fewer samples. Ninety-five percent confidence intervals (CI) for proportions were read from tables or calculated for numbers $>100[18,19]$. Absorbances were analyzed by Student's $t$ test. Survival data were analyzed by the Kaplan-Meier method and $\log$ rank test [20]. All $P$ values shown are twotailed.

\section{Results}

Specificity of HD-Ag. Among the 390 samples (including 68 cord blood samples) from children ruled uninfected, retrospective testing was positive in 12 samples (of which 3 were from cord blood), corresponding to a diagnostic specificity of 96.9\% (95\% CI, 94.8\%-98.5\%) after initial testing. No material was available for neutralization in 8 of the 12 samples initially positive. Four samples with initial readings of 0.047 , $0.028,0.028$, and 0.027 were negative by neutralization. Thus, the diagnostic specificity after neutralization of initially positive samples was 382 of 382 (100\%; 95\% CI, 99.2\%-100\%).

Diagnostic sensitivity. To determine the diagnostic sensitivity of HD-Ag and the other tests for viral components, all available results from 53 infected children were compared (table 1). When cord blood samples (in which the sensitivity of HD-Ag was $29 \%$, i.e., higher than by any other method) were excluded from the analysis, the overall diagnostic sensitivity of retrospective HD-Ag analysis was $96 \%(90.9 \%-$ 98.7\%) versus $47.7 \%(42.9 \%-52.6 \% ; P<.001)$ for UD-Ag. With respect to neutralization, the HD-Ag again compared favorably with UD-Ag: As many as 73 (97\%) of 75 samples initially positive for HD-Ag were neutralizable, while only 38 (76\%) of 50 samples positive by UD-Ag could be neutralized $(P<.004)$. Both samples initially positive by $\mathrm{HD}-\mathrm{Ag}$ that could not be neutralized were borderline $(2-3 \mathrm{pg} / \mathrm{mL})$. 
Table 1. Diagnostic sensitivity of viral parameters in samples from $53 \mathrm{HIV}$-infected children and 41 seropositive mothers.

\begin{tabular}{lcccc}
\hline Sample source & HD-Ag & UD-Ag & DNA PCR & Viral culture \\
\hline $\begin{array}{l}\text { Cord blood } \\
\text { Children by age (months) }\end{array}$ & $17,5(29)$ & $18, I(6)$ & $7,1(14)$ & $12,1(8)$ \\
$\quad 1-2$ & $3,3(100)$ & $4,0(0)$ & $3,3(100)$ & $4,4(100)$ \\
$3-4$ & $14,13(93)$ & $14,9(64)$ & $5,5(100)$ & $6,5(83)$ \\
$5-6$ & $8,7(88)$ & $10,5(50)$ & $3,3(100)$ & $4,2(50)$ \\
$7-12$ & $19,18(95)$ & $22,9(43)$ & $8,7(88)$ & $11,8(73)$ \\
$>12$ & $81,79(98)$ & $103,50(49)$ & $60,58(97)$ & $58,45(78)$ \\
Postnatal & $125,120(96.0)$ & $153,73(47.7)$ & $79,76(96.2)$ & $83,64(77.1)$ \\
Maternal & $41,9(22)$ & $41,2(4.9)$ & ND & ND \\
\hline
\end{tabular}

NOTE. Data are no. tested, no. positive (\%). HD-Ag, heat-denatured antigen; UD-Ag, undenatured antigen; DNA PCR. polymerase chain reaction for viral DNA: ND, not done.

Remarkably, in contrast to the high prevalence of $\mathrm{HD}-\mathrm{Ag}$ in children, antigenemia in delivering mothers was considerably less frequent: Only 9 of 41 maternal sera were positive (22\%; CI, 10.6\%-37.6\%; $P<.001$ ), and antigen concentrations ranged from 2 to $14 \mathrm{pg} / \mathrm{mL}$. A similar difference was found with respect to UD-Ag. Thus, infected children were significantly more frequently antigen-positive than were their mothers.

PCR for viral DNA, with $96.2 \%$ (89.4\%-99.2\%) positive postnatal samples, had sensitivity similar to $\mathrm{HD}-\mathrm{Ag}$. In addition, 1 sample was positive in duplicate with primers from the long terminal repeat region of the viral genome but negative for the gag primers and was thus ruled indeterminate (see Methods). The sensitivity of virus culture was $77.1 \%$ (66.7\%-85.3\%) in postnatal samples, which was distinctly inferior to that of $\mathrm{HD}-\mathrm{Ag}(P<.005)$.

In the prospective testing of 113 pediatric samples, 108 (95.6\%) had concordant results for both HD-Ag and PCR ( 20 positives, 88 negatives). Among the 5 samples with discordant results, PCR was correctly positive in 2 samples from infected children aged 111 and 31 months, respectively; by HD-Ag, I was just below cutoff and 1 was negative. Two $\mathrm{PCR}$-indeterminate and $\mathrm{HD}-\mathrm{Ag}$-negative samples from children aged 3 and 16 months, respectively, were probably from uninfected infants, since $\geqslant 2$ additional samples were negative by both HD-Ag and PCR analysis. The fifth discordant sample, from cord blood, was positive in screening for HD-Ag, but negative by PCR. No plasma was left for neutralization, and a fresh sample from this child has not been obtained.

Taken together, the data from both the retrospective and prospective studies demonstrate that the diagnostic sensitivities of PCR for viral DNA and HD-Ag in pediatric samples were virtually identical, even when obtained from children at early ages.

Antigenemia in mother-infant pairs. Five of 17 cord blood samples from infected infants in the retrospective study (29\%; CI, 10.3\%-56.0\%) were positive for HD-Ag (table 1). For 4 of these cases, maternal serum obtained at delivery was also available for testing. Comparisons of the absorbances of these 4 cord blood/maternal blood pairs $(0.187 / 0.009,0.113 / 0.115,0.039 / 0.027$, and $0.021 / 0.004)$ showed that none of the mothers had sufficiently high concentrations of antigen to account for that in the cord blood and that, therefore, maternal antigenemia could not be the direct cause of the cord blood antigenemia. Thus, neither intrapartum transmission of antigen nor postpartum contamination of the cord blood sample by maternal blood could account for the antigen detected in the cord blood.

Of 41 samples obtained from mothers at delivery, only 9 (22\%) were positive. Low antigen absorbances ranged from 0.020 to 0.115 (corresponding to $2.1-14.2 \mathrm{pg} / \mathrm{mL}$; mean, 0.037 . Follow-up indicated that $4(44 \%)$ of these women's 9 infants were infected; 2 of these had HD-Ag-positive cord blood. Only 6 (19\%) of the 32 infants born to HD-Ag-negative mothers were infected; the lower rate of transmission, however, was not significantly different. Two of the 6 infants were positive for $\mathrm{HD}-\mathrm{Ag}$ in cord blood while 4 were negative. In conclusion, $10(24 \%)$ of the 41 infants were infected and 4 (40\%) were $\mathrm{HD}-\mathrm{Ag}$-positive in cord blood. There was no indication that these 4 had a worse prognosis than those who were negative. Their mean survival time was 25.8 months compared with 17.5 months for those who were antigennegative; their mean symptom-free time was 12.5 versus 14.3 months. One of the 4 antigen-positive infants died at age 8 months. The other 3 are alive; 1 (child with the highest antigen concentration) without symptoms at age 36 months and 2 with symptoms at ages 19 and 40 months. Of the 6 antigen-negative infants, 3 died at 7, 10, and 20 months; 2 are alive without symptoms at ages 25 and 34 months, and 1 is alive with symptoms at age 9 months. Figure 1 shows the postnatal course of antigenemia in 6 of the 10 infected infants for whom follow-up data were available. There was no indication that infants with cord blood antigenemia had a 


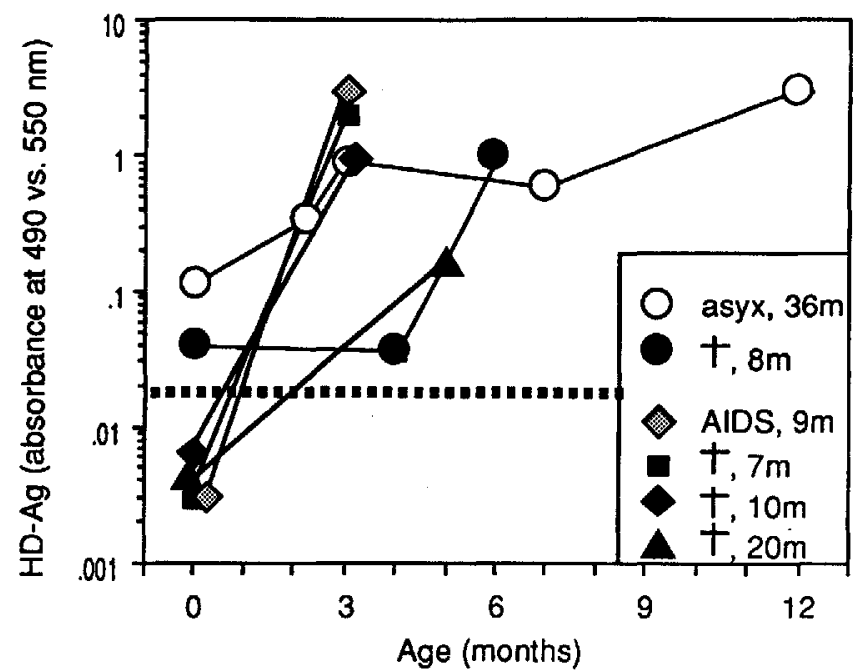

Figure 1. Postnatal follow-up of infected infants positive or negative for heat-denatured antigen (HD-Ag) in cord blood plasma. Broken line, cutoff of positivity. asyx, asymptomatic; $m$, months.

more rapid postnatal increase in antigen concentrations than those who were negative.

$H D-A g$ as a quantitative marker of virus expression. Individual quantitative results of HD-Ag testing of infected children, as expressed by absorbance values, are shown in figure 2 . None of these children received antiretroviral treatment. Thus, the measurements represent the natural course of infection. The levels of antigen were lowest in cord blood (mean absorbance $=0.037$; i.e., $\sim 4 \mathrm{pg} / \mathrm{mL}$ ). The mean level rose to a peak of $0.859(85 \mathrm{pg} / \mathrm{mL})$ in samples taken during the first 3 months after birth and was subsequently within a range of $\sim 40-150 \mathrm{pg} / \mathrm{mL}$. Thus, the mean antigen level of the infants was $>20$ times higher than that of their mothers at delivery $(P=.018)$.

Figure $2 \mathrm{~A}$ shows levels of $\mathrm{HD}-\mathrm{Ag}$ in dependence of time and the clinical status of 36 infected children who had $\geqslant 2$ samples tested. After exclusion of cord blood samples, symptomatic children had, with a mean absorbance of 0.918 , antigen levels roughly twice as high as children without symptoms (mean, $0.497 ; P=.014$ ). The difference was largely due to a difference in levels found during the first 2 years (mean, 1.119 vs. $0.407 ; P=.004$ ). After age 2 years, the difference was no longer significant. Symptomatic children $>2$ years old had antigen concentrations similar to those without symptoms.

Individual courses of antigenemia are shown in figure 2B. In general, a rapid increase in antigen level during the first few months of life was found. In some instances, a subsequent decrease was observed. In others, the antigen values remained relatively stable or increased further. Typically, the antigen levels remained above an absorbance of 0.100 (corresponding to $\sim 10 \mathrm{pg} / \mathrm{mL}$ ), being relatively stable in some children but showing considerable fluctuation in others.

Prognostic relevance of levels of $\mathrm{HD}-\mathrm{Ag}$. Levels of $\mathrm{HD}-\mathrm{Ag}$ in cord blood, at ages 1-6 months, and at 7-12 months were compared with survival time. The levels of HD-Ag in cord blood or in infants at 7-12 months were of no prognostic value (data not shown). By contrast, antigen levels in 20 infected infants within 6 months after birth were inversely associated with survival time (figure 3 ). Four of 5 infants in whom an absorbance $\geqslant 0.900$ was measured in this period died at $5,7,10$, and 18 months; 1 is alive with symptoms at age 20 months. By contrast, only 4 (27\%) of 15 infants with absorbance values $<0.900$ have died (at ages 5, 8, 20, and 26

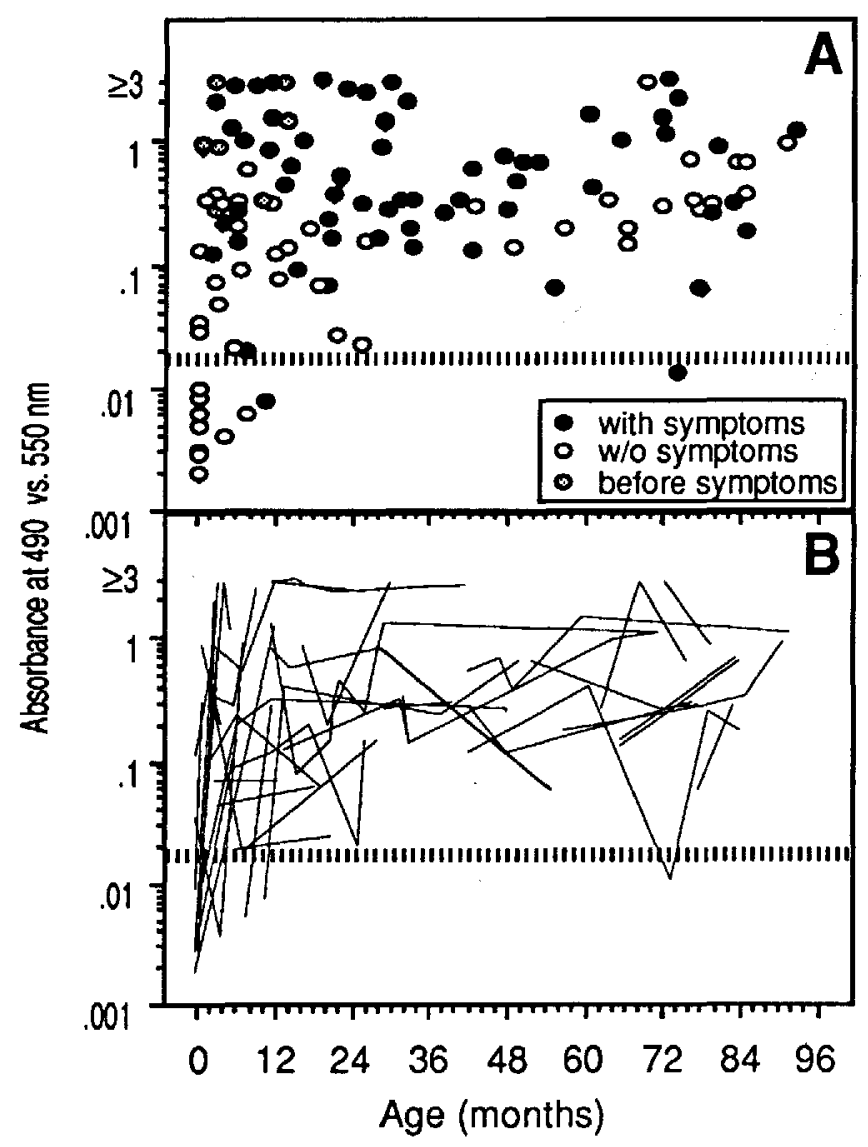

Figure 2. Age-dependent concentrations of heat-denatured antigen determined by absorbance values in infected children from whom $\geqslant 2$ samples could be tested. Cutoff (broken bar) corresponds to $\sim 2 \mathrm{pg} / \mathrm{mL}$. Absorbances of 0.100 and 1.000 correspond to $\sim 10$ and $\sim 100 \mathrm{pg} / \mathrm{mL}$, respectively. Values above detection range of EIA reader were recorded as 3.000. A, Antigen in dependence of age and clinical status. Figure differentiates between patients asymptomatic (CDC P-1) at testing, patients who were still asymptomatic but developed symptoms before next sample was taken, and symptomatic patients (CDC P-2). B, Individual courses of antigenemia. Results of all samples from a patient are connected by line. w/o, without. 


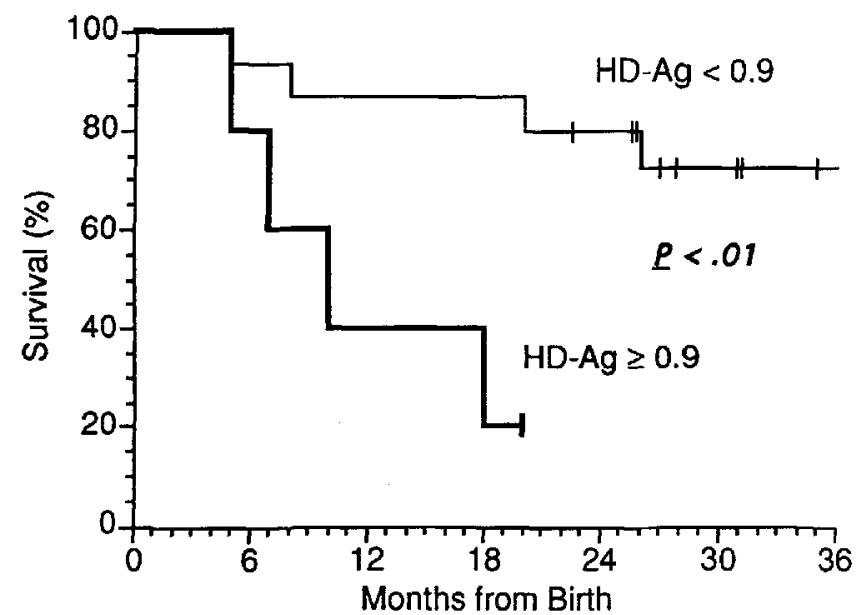

Figure 3. Prognostic significance of heat-denatured antigen (HD-Ag) measured during first 6 months of life. Survival time of 20 children is shown in relation to HD-Ag level in Kaplan-Meier analysis. Five children who had $\geqslant 1$ sample with absorbance $\geqslant 0.900$ were compared with 15 children whose samples had absorbances $<0.900$. Infants with absorbances $<0.900$ had significantly longer survival (log rank test: $\chi^{2}=7.28, P<.01$ ).

months; 11 remain alive (range, $22-77$ months). Statistical analysis indicated that the survival times of the two groups differed significantly $(P<.01)$.

\section{Discussion}

The World Health Organization estimates that 10 million children will have been infected with HIV by the year 2000 . Most will be born in economically poor countries [21]. Thus, recognition and treatment of pediatric HIV infection are of increasing importance and depend on sensitive yet affordable methods.

Recent studies in which acidification was used for immune complex dissociation demonstrated that testing for antigen may identify cases of pediatric HIV-I infection within the first few weeks of life or even at birth $[11,22]$. We have now found a simple single-step procedure for immune complex dissociation that does not require chemical reagents. Using this procedure for retrospective and prospective analysis of a cohort of pediatric samples, we found that HD-Ag had the same diagnostic sensitivity as PCR, which is considered the most sensitive test for HIV [1, 23-26]. Unfortunately, since few samples taken during the first few weeks of life were available, we could not adequately assess the efficacy of the method in the neonatal period. However, on the basis of the low detection level $(2 \mathrm{pg} / \mathrm{mL})$ and the finding that HD-Ag yielded more positive findings among cord blood samples from infected children than PCR, even though our PCR procedure can detect a single copy of HIV-1 DNA [17], we predict that the test, when done in the early weeks of life, will be as sensitive as PCR. Detection of HD-Ag was also highly specific, as demonstrated by successful neutralization in $97 \%$ of positive samples from infected children and by the fact that the rare cases of initially positive results in samples from children ruled uninfected were negative when subjected to neutralization.

The finding of positivity for HD-Ag in $\sim 30 \%$ of cord blood samples from infected infants is in accordance with results obtained in other studies that used PCR or viral culture in samples obtained in the first week of life [1]. These data suggest that mother-to-infant transmission of HIV-l occurs in at least one-third of cases in utero. In contrast, our antigen data do not support the observation seen in investigations using DNA PCR that infants in whom infection is demonstrated at birth have a more rapid clinical course than those in whom it is detected later [27]. This discrepancy may relate to the fact that different viral components, which may not be strictly related to each other with regard to development of disease, are recognized by these two tests. Positivity by DNA PCR indicates that a small fraction of PBMC (i.e., $\geqslant 1-10 / 100,000$ cells) is infected. Recent findings in adults indicate that in the early stage of infection, the virus is predominantly localized in lymphoid tissues $[28,29]$. The presence, at sufficient concentration for detection by PCR, of HIV-infected cells in umbilical cord blood might thus represent a more advanced stage of infection than the presence of immune-complexed viral antigen, which may originate in the lymphatic foci of infection.

Our finding that the level of antigen during the first 6 months of life is of prognostic significance is of clinical interest (figure 3). Since the number of cases is small, it will be necessary to confirm this association in larger studies. However, these data suggest that testing for HD-Ag might be used for early diagnosis of pediatric HIV infection and identification of infants who may benefit from early antiretroviral treatment. Furthermore, this prognostic association suggests that events early in the course of infection influence the clinical course of pediatric HIV infection. Whether these events are due to viral or host factors or both remains to be elucidated.

In contrast, the presence of HD-Ag in cord blood (figure 1) or after 6 months of life did not correlate with prognosis nor were antigen levels in children $>2$ years old significantly associated with the presence of symptoms (figure 2). The persistent antigenemia found in virtually all children contrasted with the low prevalence of HD-Ag and the low concentrations found in mothers at delivery. Adult HIV-1 infection is characterized by an initial peak of viral replication early after infection, which is followed by a period during which little virus or antigen is present in plasma $[7,30,31]$. In our previous study of samples from 50 infected adults [12], antigen was found in only 7 (35\%) of 20 samples that had high titers of antibody to $\mathrm{p} 24$, a condition associated 
with an early stage of infection and a more favorable prognosis $[4,32,33]$. The prevalence and levels of HD-Ag in the mothers are in accordance with these findings. The fact that the asymptomatic infants, despite being infected with viruses carried by their mothers, had continuously detectable antigenemia suggests that, unlike that of adults, an infant's immune system is not capable of controlling HIV expression. This may represent one of the factors responsible for the more rapid progression of pediatric HIV infection.

In conclusion, $\mathrm{HD}-\mathrm{Ag}$ is a sensitive, specific, and quantitative marker for HIV-I in pediatric infection. These properties, together with other advantages of EIAs, such as simplicity, assay speed, the possibility of automatization, and relatively low cost, render testing for $\mathrm{HD}-\mathrm{Ag}$ an attractive alternative to the technically demanding and expensive PCR or time-consuming viral cultivation. The systematic use of $\mathrm{HD}-\mathrm{Ag}$ in prospective and retrospective studies larger than ours should contribute to earlier diagnosis, identification of children with unfavorable prognosis, and to better knowledge of quantitative viral aspects in the pathogenesis of pediatric AIDS.

\section{Acknowledgments}

We thank Dora Pontelli, Sonja Kreiner, Annemieke Münür, Antonietta Baumgartner, Lucia Porong, and André Fitsche for valuable technical assistance.

\section{Swiss Neonatal HIV Study Group}

D. Nadal, Children's University Hospital, Zurich; C.-A. Wyler, Department of Pediatrics, University of Geneva; A. Calame, Department of Pediatrics, University of Lausanne; U. B. Schaad, formerly, Department of Pediatrics, University of (Bernenow: Department of Pediatrics, University of Basel); H. P. Gnehm, Children's Hospital, Aarau; J. Klingler, Children's Hospital, Biel; M. P. Gianinazzi, Children's Hospital, Lugano; G. Schubiger, Children's Hospital, Lucern; Ch. Baumgartner, Children's Hospital, St. Gallen; H. Kuchler, Children's Hospital, Sion; U. Hunziker, Children's Hospital, Winterthur; U. Bühlmann, Children's Hospital, Stadtspital Triemli, Zurich; C. Kind, St. Gallen (study coordinator).

\section{References}

1. Report of a consensus workshop, Siena, Italy, January 17-18, 1992. Early diagnosis of HIV infection in infants. J AIDS 1992;5:1169-78.

2. Ellaurie M, Calvelli TA, Rubinstein A. Human immunodeficiency virus (HIV) circulating immune complexes in infected children. AIDS Res Hum Retroviruses 1990;6:1437-41.

3. Pedersen C, Moller-Nielsen C, Vestergaard BF, Gerstoft J, Korgsgaard K. Nielsen JQ. Temporal relation of anigenaemia and loss of antibod- ies to core antigens to development of clinical disease in HIV infection. Br Med J 1987;295:567-9.

4. Lange JM, Paul DA, de Wolf F, Coutinho RA, Goudsmit J. Viral gene expression, antibody production and immune complex formation in human immunodeficiency virus infection. AIDS 1987;1:15-20.

5. Kageyama S, Yamada O, Mohammad SS, et al. An improved method for the detection of HIV antigen in the blood of carriers. J Virol Methods 1988;22:125-31.

6. Mathiesen T, Sundqvist VA, Albert J, Ohlsson E, Wahren B. Acid-hydrolysis of serum samples to increase detection of HIV antigen. $J$ Virol Methods 1988;22:143-8.

7. Von Sydow M, Gaines H, Sönnerborg A, Forsgrean M, Pherson PO, Strannegard $\mathrm{O}$. Antigen detection in primary HIV infection. BMJ 1988;296:238-40.

8. Kestens L, Hoofd G, Gigase PL, Deleys R, van der Groen G. HIV antigen detection in circulating immune complexes. J Virol Methods 1991;31:67-76.

9. Nishanian P, Huskins KR, Stehn S, Detels R, Fahey JL. A simple method for improved assay demonstrates that HIV p24 antigen is present as immune complexes in most sera from HIV-infected individuals. J Infect Dis 1990;162:21-8.

10. Ascher DP, Roberts C, Fowler A. Acidification modified p24 antigen capture assay in HIV seropositives. J AIDS 1992;5:1080-3.

11. Miles SA, Balden E, Magpantay L, et al. Rapid serologic testing with immune-complex-dissociated HIV p24 antigen for early detection of HIV infection in neonates. N Engl J Med 1993;328:297-302.

12. Schüpbach J, Böni J. Quantitative and sensitive detection of immunecomplexed and free HIV antigen after boiling of serum. $J$ Virol Methods 1993:43:247-56.

13. Kind C, Brändle B, Wyler CA, et al. Epidemiology of vertically transmitted HIV-1 infection in Switzerland: results of a nationwide prospective study. Eur J Pediatr 1992;151:442-8.

14. Centers for Disease Control. Classification system for human immunodeficiency virus (HIV) infection in children under 13 years of age. MMWR 1987;36:225-36.

15. Association of State and Territorial Public Health Laboratory Directors and AIDS Program and Centers for Disease Control. Interpretation and use of the Western blot assay for serodiagnosis of human immunodeficiency virus type 1 infections. MMWR 1989;38:1-7.

16. Ho DD, Moudgil T, Alam M. Quantitation of human immunodeficiency virus type 1 in the blood of infected persons. N Engl J Med 1989;321:1621-5.

17. Böni J, Schüpbach J. Primer extension analysis provides a sensitive tool for the identification of PCR-amplified DNA from HIV-1. J Virol Methods 1993;42:309-22.

18. Wissenschaftliche Tabellen Geigy. 8th ed. Vol 3. Basel, Switzerland: Ciba-Geigy, 1980:89-102

19. Sachs L. Angewandte Statistik. 5th ed. New York: Springer-Verlag, 1978:258-9.

20. Cox DR, Oakes D. Analysis of survival data. London: Chapman and Hall, 1984.

21. Chin J. Current and future dimensions of the HIV/AIDS pandemic in women and children. Lancet 1991;336:221-4.

22. Luzuriaga K, McQuilken P, Alimenti A, Somasundaran M, Hesselton RA, Sullivan JL. Early viremia and immune responses in vertical human immunodeficiency virus type 1 infection. $J$ Infect Dis 1993; 167:1008-13.

23. Borkowsky W, Krasinski K, Pollack H, Hoover W, Kaul A, IlmetMoore T. Early diagnosis of human immunodeficiency virus infection in children $<6$ months of age: comparison of polymerase chain reaction, culture, and plasma antigen capture techniques. J Infect Dis 1992;166:616-9.

24. Comeau AM, Harris JA, McIntosh K, Weiblen BJ, Hoff R, Grady GF. 
Polymerase chain reaction in detecting HIV infection among seropositive infants: relation to clinical status and age and to results of other assays, J AIDS 1992;5:271-8.

25. Brandt CD. Rakusan TA, Sison AV, et al. Detection of human immunodeficiency virus type $\mathbf{I}$ infection in young pediatric patients by using polymerase chain reaction and biotinylated probes. J Clin Microbiol 1992;30:36-40.

26. Krivine A. Firtion G, Cao L, Francoual C, Henrion R, Lebon P. HIV replication during the first weeks of life. Lancet 1992;339:1187-9.

27. Rogers MF, Ou CY, Rayfield $M$, et al. Use of the polymerase chain reaction for early detection of the proviral sequences of human immunodeficiency virus in infants born to seropositive mothers. $\mathrm{N}$ Engl $\mathrm{J}$ Med 1989;320: 1649-54.

28. Pantaleo G. Graziosi C. Demarest JF, et al. HIV infection is active and progressive in lymphoid tissue during the clinically latent stage of disease. Nature 1993;362:355-8.
29. Embretson J, Zupancic M, Ribas JL, et al. Massive covert infection of helper $\mathrm{T}$ lymphocytes and macrophages by HIV during the incubation period of AIDS. Nature 1993;362:359-62.

30. Daar ES, Moudgil T, Meyer RD, Ho DD. Transient high levels of viremia in patients with primary human immunodeficiency virus type I infection. N Engl J Med 1991;324:96 1-4.

31. Clark SJ, Saag MS, Decker WD, et al. High titers of cytopathic virus in plasma of patients with symptomatic primary HIV-I infection. $\mathrm{N}$ Engl J Med 1991;324:954-60.

32. Schüpbach J, Haller $O$, Vogt M, et al. Antibodies to HTLV-III in Swiss patients with AIDS, pre-AIDS, and in groups at risk for AIDS. N Engl J Med 1985;312:265-70.

33. Weber JN, Clapham PR, Weiss RA, et al. Human immunodeficiency virus infection in two cohorts of homosexual men: neutralising sera and association of anti-gag antibody with prognosis. Lancet $1987 ; 1: 119-22$. 